\title{
Sclerosing encapsulating peritonitis as a rare cause of intestinal obstruction after the treatment of peritoneal mesothelioma: a case report and review of the literature
}

\author{
Zhizhan Ni ${ }^{1 \#}$, Qing Chen ${ }^{2 \#}$, Chenshen Huang ${ }^{1 \#}$, Song Wang ${ }^{1}$, Qi Huang ${ }^{1}$ \\ ${ }^{1}$ Department of General Surgery, Tongji Hospital, Tongji University School of Medicine, Shanghai, China; ${ }^{2}$ Department of General Surgery, \\ Shanghai Tenth People's Hospital, Tongji University School of Medicine, Shanghai, China \\ \#These authors contributed equally to this work. \\ Correspondence to: Qi Huang. Department of General Surgery, Tongji Hospital, Tongji University School of Medicine, 389 Xincun Road, Shanghai \\ 200065, China. Email: hqhq0078@163.com.
}

\begin{abstract}
Sclerosing encapsulating peritonitis (SEP) is a rare cause of intestinal obstruction that is characterized by a thick greyish-white fibrotic membrane encasing the small bowel. The clinical features are typically nonspecific, and they occasionally present with recurrent episodes of incomplete or complete intestinal obstruction. The etiology of SEP remains unclear, and the diagnosis is often delayed mainly due to the lack of specific symptoms. Here, we first report a patient who suffered from SEP after treatment for malignant mesothelioma (MM) with tumor resection supplemented with hyperthermic intraperitoneal chemotherapy (HIPEC) once and intraperitoneal chemotherapy (IPC) eight times. The patient was discharged ten days after surgery and was free of complications at the 4-month follow-up. In addition, we reviewed the published literature from PubMed, only 7 articles of 16 cases finally met the defined requirements. Nine cases of SEP after IPC and 7 cases of SEP after HIPEC were previously reported. We synthetically review the pathogenesis, treatments, and outcomes. In conclusion, SEP is a rare abdominal disease, which is difficult to diagnose preoperatively. CT scanning is the most helpful imaging method for the diagnosis of SEP. Surgery is the most effective method for diagnosis and treatment, if conservative treatment has no effect or abdominal symptoms are aggravated.
\end{abstract}

Keywords: Sclerosing encapsulating peritonitis (SEP); malignant mesothelioma (MM); intraperitoneal chemotherapy (IPC); case report

Submitted Nov 14, 2020. Accepted for publication Mar 12, 2021.

doi: $10.21037 /$ tcr-20-3259

View this article at: https://dx.doi.org/10.21037/tcr-20-3259

\section{Introduction}

Sclerosing encapsulating peritonitis (SEP) is a rare clinical syndrome characterized by an inflammatory fibrocollagenous membrane, and segmental intestines are often encapsulated within (1). The clinical features are typically nonspecific, and they occasionally present with recurrent episodes of incomplete or complete intestinal obstruction (2). Early-stage symptoms of the disease include fever, weight loss, constipation, abdominal distention, and nausea. However, as the disease progresses, thickened membranes can encapsulate the intestines, and the signs of intestinal obstruction become more obvious $(2,3)$. Although the factors that lead to SEP remain to be fully elucidated, intraperitoneal chemotherapy (IPC) is widely reported to be a promoter of disease progression $(4,5)$. Here, we first report a patient who suffered from SEP after treatment of malignant mesothelioma (MM) with tumour resection supplemented with hyperthermic intraperitoneal chemotherapy (HIPEC) once and IPC eight times. We present the following article in accordance with the CARE reporting checklist (available at https://dx.doi.org/10.21037/ tcr-20-3259). 

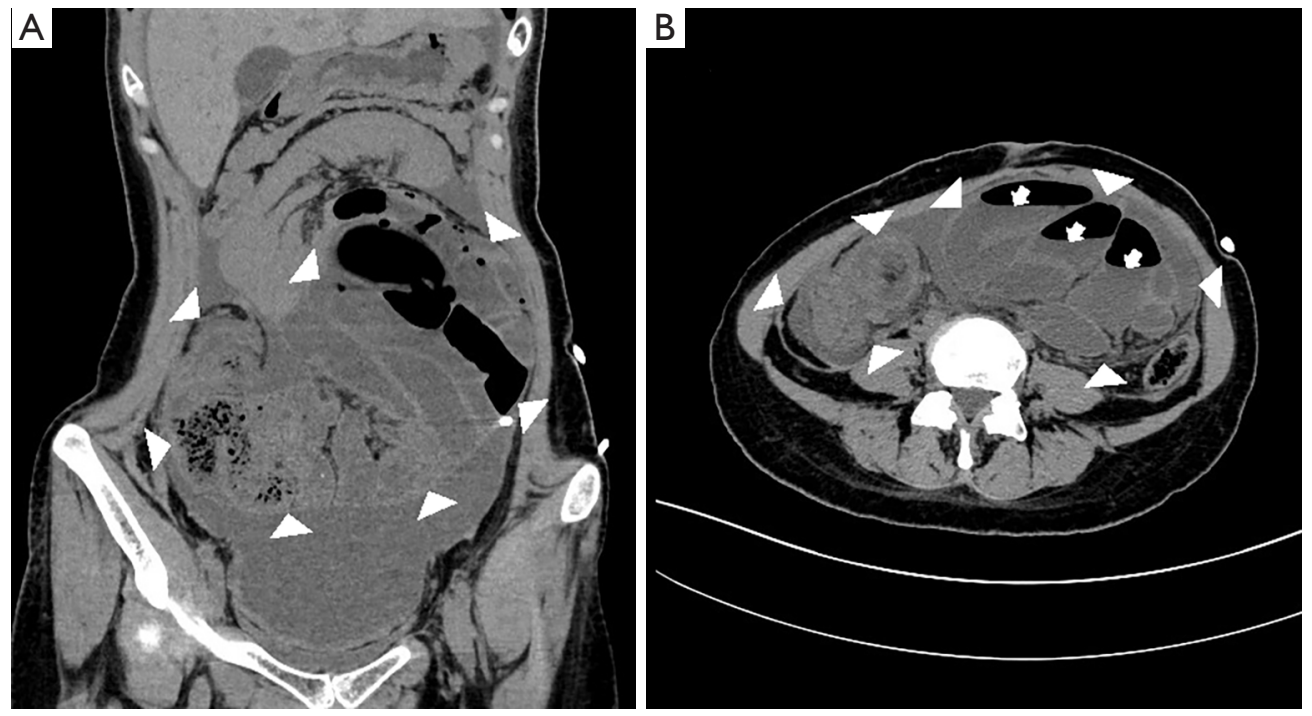

Figure 1 Abdominal computed tomography (CT) of sclerosing encapsulating peritonitis (SEP) in longitudinal (A) and transverse (B) sections. Clumped small intestine loops were surrounded and confined within a membranous cavity. Membrane boundaries are marked by white triangles. Dilated small intestines with multiple air-fluid levels (marked by arrows) were observed.

\section{Case presentation}

A 32-year-old woman presented in January 2019 with acute crampy abdominal pain and other signs of acute small bowel obstruction, including distention, vomiting and constipation. No fever or other symptoms were present. Medical history indicated MM resection at 28 and postoperative HIPEC with cisplatin at a dose of $600 \mathrm{mg}$ once supplemented by IPC with cisplatin at a dose of $50 \mathrm{mg}$ and intravenous chemotherapy with pemetrexed at a dose of $800 \mathrm{mg}$ eight times. She experienced several episodes of incomplete bowel obstruction since MM resection and was relieved through conservative treatment.

On examination, marked abdominal distension and a mass in the central abdomen were found. Laboratory blood tests showed leucocytosis and elevated C-reactive protein (CRP) levels. X-ray tests showed dilation of small intestine loops, intestinal fluid retention and multiple air-fluid levels (data not shown). Abdominal computed tomography (CT) scanning revealed that clumped small intestine loops were surrounded and confined within a membranous cavity (Figure 1A). Transverse CT scanning sections showed dilated small intestines with multiple air-fluid levels that were enwrapped and isolated by irregularly shaped membranous tissues (Figure 1B).

The decision was made to perform an exploratory laparotomy due to obstinate small bowel obstruction. During the surgery, we found complete encapsulation of the proximal jejunum to the distal ileum by prominently thickened fibrous membranes (Figure $2 A, B$ ). SEP was confirmed intraoperatively, and meticulous adhesiolysis was performed. All the adhesions and cocoon membranes were released by blunt or sharp separation. The entire intestines were completely released without causing any secondary damage, and encapsulating peritonea were partially resected and sent for histological examination (Figure 2C). Symptoms of small bowel obstruction were relieved, and bowel movements were recovered at day 9 postoperationally. The patient was free of complications with normal bowel function at the 4-month follow-up.

The resected abdominal cocoon (AC) was histologically processed by hematoxylin and eosin staining ( 5 minutes each in room temperature). Histological examination of the resected membrane showed high proliferation of fibroconnective tissue associated with peritoneal mesothelioma (Figure $3 A, B$ ).

All procedures performed in studies involving human participants were in accordance with the ethical standards of the institutional and/or national research committee(s) and with the Helsinki Declaration (as revised in 2013). Written informed consent was obtained from the patient.

\section{Methods}

The literature search was conducted using the PubMed 

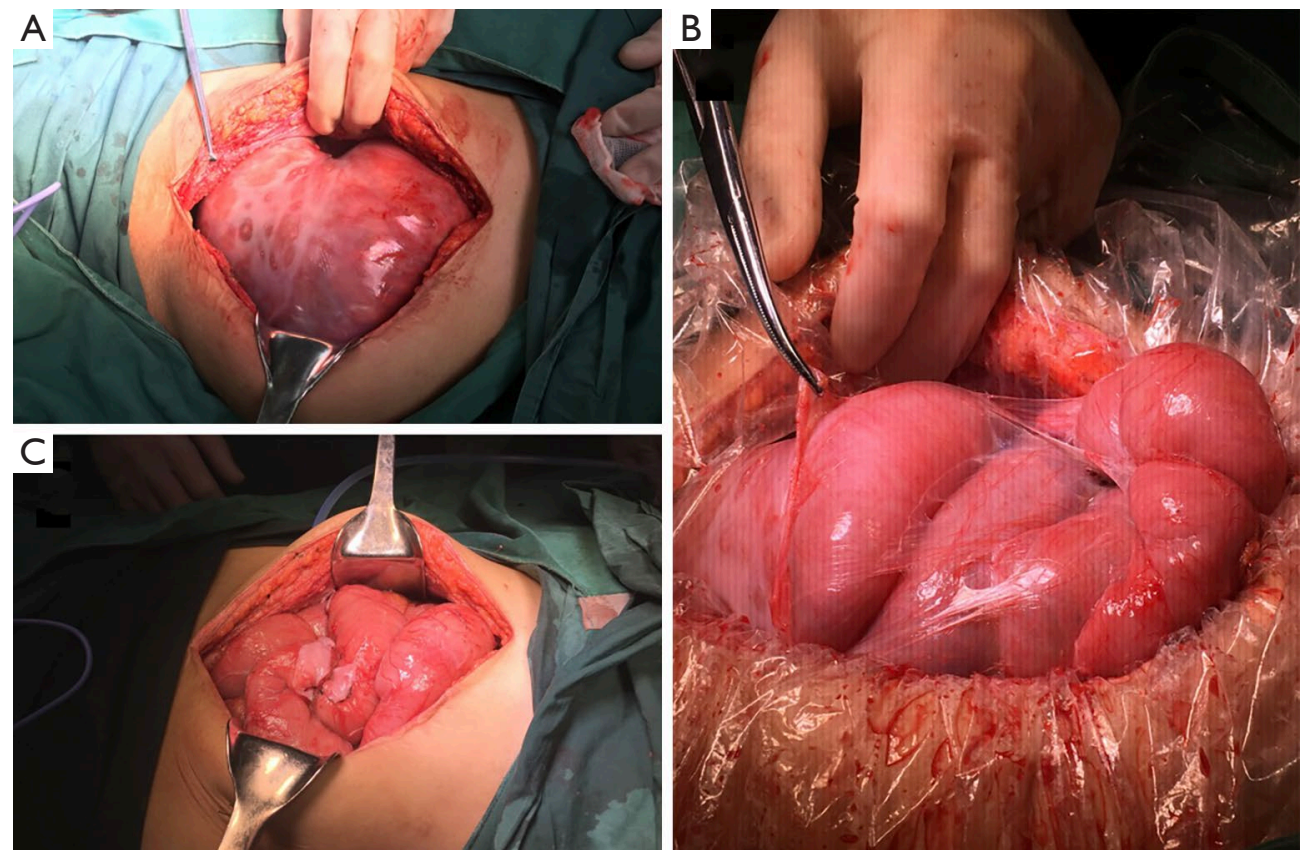

Figure 2 Intraoperative photographs of encapsulated small intestine. (A,B) Complete encapsulation of the upper jejunum and the ileocecal small intestine by prominently thickened fibrous membranes was detected. (C) The encapsulated intestinal loops were completely released in surgery.
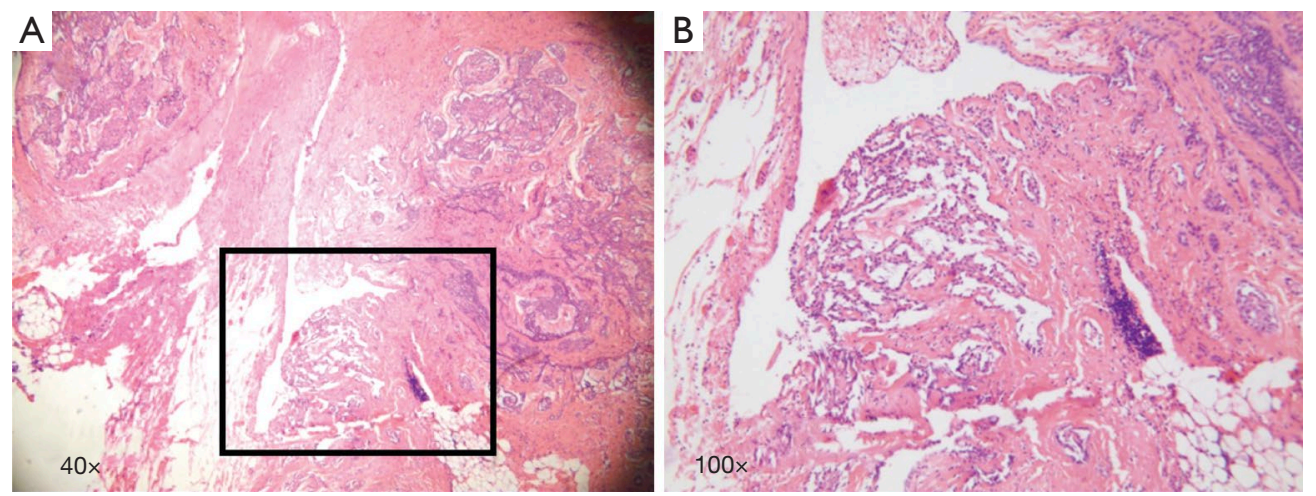

Figure 3 The paraffin-embedded tissue sections were used for examination of hematoxylin and eosin (HE) staining. Pathological examination of resected membranous tissue revealed high proliferation of fibroconnective tissue along with peritoneal mesothelioma tissue.

database up to December 2019 with the following index words: "abdominal cocoon"[Title] OR "sclerosing encapsulating peritonitis"[Title] OR "encapsulating peritoneal sclerosis"[Title] OR "peritoneal fibrosis"[Title] OR "intraperitoneal chemotherapy" [Title]. The search only included case reports, and the search was limited to papers in the English language.

\section{Results}

Of the 2,790 papers found, only 465 papers were included as case reports. Seven articles of 16 cases finally met the defined requirements of SEP after IPC or HIPEC treated by the surgical approach. Nine cases of SEP after IPC and 7 cases of SEP after HIPEC were previously reported and are summarized in Table 1 (4,6-11). 
Table 1 Reported cases of sclerosing encapsulating peritonitis after intraperitoneal chemotherapy treated by the surgical approach

\begin{tabular}{|c|c|c|c|c|c|c|c|c|}
\hline No & Author & Years & Primary Disease & Chemotherapy & Sex & IP/HIPEC & Treatment & SEP after initial surgery \\
\hline 2 & Braly & 1986 & Ovarian cancer & Cisplatin, 5-FU & Female & IP & Laparotomy & Unknown \\
\hline 3 & Braly & 1986 & Ovarian cancer & Cisplatin, 5-FU & Female & IP & Laparotomy & Unknown \\
\hline 4 & Vlasveld & 1992 & Mesothelioma & Metoxantrone & Male & IP & Surgery & Alive, 48 months \\
\hline 6 & Atiq & 1993 & Gastric cancer & Cisplatin, 5-FU & Unknown & IP & Surgery & Unknown \\
\hline 7 & Atiq & 1993 & Gastric cancer & Cisplatin, 5-FU & Unknown & IP & Surgery & Unknown \\
\hline 8 & Atiq & 1993 & Gastric cancer & Cisplatin, 5-FU & Unknown & IP & Surgery & Unknown \\
\hline 9 & Atiq & 1993 & Gastric cancer & Cisplatin, 5-FU & Unknown & IP & Laparotomy & Unknown \\
\hline 12 & Gabriel & 2018 & Appendiceal DPAM & Oxaliplatin & Female & HIPEC & Laparotomy & Alive, 27 months \\
\hline 13 & Gabriel & 2018 & Colon Adenocarcinoma & Oxaliplatin & Male & HIPEC & Laparotomy & Alive, 4 months \\
\hline 14 & Gabriel & 2018 & Cystic mesothelioma & $\begin{array}{l}\text { Cisplatin, Doxorubicin, } \\
\text { Ifosfamide, MESNA }\end{array}$ & Male & HIPEC & Surgery & Alive, 6 years \\
\hline 15 & Gabriel & 2018 & Appendiceal DPAM & $\begin{array}{l}\text { Mitomycin C, Doxorubicin, } \\
\text { 5-FU, Leucovorin }\end{array}$ & Female & HIPEC & Surgery & Alive, 6 years \\
\hline 16 & Clodagh & 2018 & Colon cancer & $\begin{array}{l}\text { Oxaliplatin, 5-FU, } \\
\text { Mitomycin C }\end{array}$ & Male & HIPEC & Laparotomy & Unknown \\
\hline
\end{tabular}

IP, intraperitoneal perfusion chemotherapy; HIPEC, hyperthermic intraperitoneal chemotherapy; 5-FU, 5-fluorouracil; DPAM, disseminated peritoneal adenomucinosis; MESNA, 2-mercaptoethanesulfonate sodium.

\section{Discussion}

SEP is a rare clinical syndrome characterized by an inflammatory fibro-collagenous membrane, and segmental intestines are often encapsulated within, leading to recurrent small bowel obstruction (1). Although the aetiology still requires further investigation, SEP is commonly classified as primary (idiopathic) and secondary (12). Primary SEP, which is also known as abdominal cocoon (AC), was previously reported in adolescent females with fallopian tube inflammation or irregular menstruation presumably due to chemical peritonitis induced by retrograde infection (13). Other causative diseases of primary SEP, such as hereditary omentum heteroplasia (14) and peritoneal bacterial infection (15), have also been reported. Secondary SEP, which is presented more commonly in previous reports, develops when a local or systemic factor triggers inflammatory cascades in the peritoneum (12). SEP is most commonly triggered by long-term peritoneal dialysis (PD) $(16,17)$ but is also reported to be associated with other factors, including medications $(18,19)$, infections $(20,21)$, abdominal tumour surgeries and postoperative chemotherapy $(4,5,9)$, peritoneal shunts (22), recurrent episodes of peritonitis (23) and autoimmune disorders $(24,25)$. However, the pathophysiology of these various factors triggering SEP is not well studied with the exception of $\mathrm{PD}$, which is referred to as the "two-hit" hypothesis $(26,27)$. Non-inflammatory peritoneal sclerosis accumulates in repeated PD ("first hit") followed by precipitation of a pro-inflammatory cascade (transforming growth factor $\beta 1$, interleukin-6, etc.) and pro-angiogenic (vascular endothelial growth factor) cytokines ("second-hit"), leading to transdifferentiation of peritoneal mesothelial cells and formation of inflammatory fibro-collagenous membranes.

To our knowledge, IPC and HIPEC could also cause peritoneal fibrosis and repeated intestinal obstruction due to dense adhesions $(4,5,9)$. Markman et al. first suggested 
that the use of IPC would cause extensive adhesion formation in 1986 (28). In his study, 1,103 patients were treated with IPC. Two cases reported extensive sclerosis related to cisplatin. Jacquet et al. reported a retrospective study including 196 patients treated with at least 1 cycle of early postoperative IPC with 5-fluorouracil (5-FU) and mitomycin C (29). Among those patients, 4 developed peritoneal fibrosis due to dense adhesions. According to the "two-hit" theory in PD patients, peritoneal sclerosis and fibrosis formation resulting from IPC can be considered the "first hit". Additional pro-inflammatory inducing incidents would be required as a "second hit" for SEP development. There are limited clinical data and reports on SEP caused by HIPEC and IPC (Table 1). The generation of SEP mostly resulted from tumours of the gynaecology and digestive system. In 9 cases of SEP after IPC, at least 1 cycle of postoperative cisplatin and 5-FU was performed. Among the 7 cases of HIPEC, 2 cases accepted oxaliplatin, and 2 cases accepted mitomycin $\mathrm{C}$ and 5-FU. To some extent, the repeated treatment of IPC is similar to that of PD for renal failure. According to the "two-hit" hypothesis, this could be a trigger for SEP.

In our case, the primary disease of SEP was MM, which is a rare disease that develops from mesothelial cells covering the surface of serous membranes (30). MM accounts for approximately $10-20 \%$ of all mesotheliomas and has an estimated incidence of 40-60 in 1,000,000 per year (31). CRS and HIPEC are the traditional treatments for MM. In previous studies, only Vlasveld reported a case of SEP caused by MM after intravenous chemotherapy with metoxantrone (11). The patient in our study received 6 cycles of intravenous chemotherapy with cisplatin and 1 additional cycle of HIPEC cisplatin. This is the first reported patient treated with both IPC and HIPEC reported to be diagnosed with SEP.

Laparotomy is always the gold-standard diagnostic approach for SEP $(2,32,33)$ and is often performed in patients who fail conservative therapy. Surgical exploration confirms the diagnosis, determines the range and severity of bowel obstruction and determines whether a segmental necrotic bowel needs to be removed (12). To reduce the possibility of intestinal injury during the perioperative period that could cause complications, such as postoperative intestinal leakage and short-intestine syndrome, adhesiolysis is often sufficient to relieve intestinal obstruction (34). In this patient, we performed meticulous adhesiolysis without membrane excision. The patient was free of uncomfortable complaints at the 4-month follow-up.

\section{Conclusions}

In conclusion, SEP is a rare abdominal disease and is difficult to diagnose preoperatively. When a patient shows the main clinical features of intestinal obstruction after HIPEC or IP similar to this patient, SEP should be considered. CT scanning is the most helpful imaging method for the diagnosis of SEP. If conservative treatment has no effect or abdominal symptoms are aggravated, surgery is the last option. Laparotomy plays an important role in its diagnosis and treatment. The aim of the operation is to release bowel encapsulation and preserve the function of the small intestines as much as possible.

\section{Acknowledgments}

We appreciate the patient's approval to present this case, and we also acknowledge the support from the Department of General Surgery, Department of Pathology, and Department of Radiology in patient diagnosis and treatment.

Funding: This study was funded by the Shanghai Science and Technology Innovation Action Plan (Grant No. 19441905700) and the Clinical Research and Cultivation Project of Shanghai Tongji Hospital [Grant No. ITJ (ZD) 1802, ITJ (ZD) 1804].

\section{Footnote}

Reporting Checklist: The authors have completed the CARE reporting checklist. Available at https://dx.doi. org/10.21037/tcr-20-3259

Conflicts of Interest: All authors have completed the ICMJE uniform disclosure form (available at https://dx.doi. org/10.21037/tcr-20-3259). The authors have no conflicts of interest to declare.

Ethical Statement: The authors are accountable for all aspects of the work in ensuring that questions related to the accuracy or integrity of any part of the work are appropriately investigated and resolved. All procedures performed in studies involving human participants were in accordance with the ethical standards of the institutional and/or national research committee(s) and with the Helsinki Declaration (as revised in 2013). Written informed consent was obtained from the patient.

Open Access Statement: This is an Open Access article 
distributed in accordance with the Creative Commons Attribution-NonCommercial-NoDerivs 4.0 International License (CC BY-NC-ND 4.0), which permits the noncommercial replication and distribution of the article with the strict proviso that no changes or edits are made and the original work is properly cited (including links to both the formal publication through the relevant DOI and the license). See: https://creativecommons.org/licenses/by-nc-nd/4.0/.

\section{References}

1. Xu P, Chen LH, Li YM. Idiopathic sclerosing encapsulating peritonitis (or abdominal cocoon): a report of 5 cases. World J Gastroenterol 2007;13:3649-51.

2. Li N, Zhu W, Li Y, et al. Surgical treatment and perioperative management of idiopathic abdominal cocoon: single-center review of 65 cases. World J Surg 2014;38:1860-7.

3. Machado NO. Sclerosing Encapsulating Peritonitis: Review. Sultan Qaboos Univ Med J 2016;16:e142-51.

4. Takebayashi K, Sonoda H, Shimizu T, et al. Successful surgical approach for a patient with encapsulating peritoneal sclerosis after hyperthermic intraperitoneal chemotherapy: a case report and literature review. BMC Surg 2014;14:57.

5. Kaman L, Iqbal J, Thenozhi S. Sclerosing encapsulating peritonitis: complication of laparoscopic cholecystectomy. J Laparoendosc Adv Surg Tech A 2010;20:253-5.

6. Braly P, Doroshow J, Hoff S. Technical aspects of intraperitoneal chemotherapy in abdominal carcinomatosis. Gynecol Oncol 1986;25:319-33.

7. Atiq OT, Kelsen DP, Shiu MH, et al. Phase II trial of postoperative adjuvant intraperitoneal cisplatin and fluorouracil and systemic fluorouracil chemotherapy in patients with resected gastric cancer. J Clin Oncol 1993;11:425-33.

8. Aihara H, Maruoka H, Kiyozaki H, et al. Sclerosing encapsulating peritonitis (SEP) as a delayed complication of continuous hyperthermic peritoneal perfusion (CHPP): Report of a case. Surg Today 2003;33:232-6.

9. Liberale G, Sugarbaker PH. Sclerosing encapsulating peritonitis as a potential complication of cytoreductive surgery and HIPEC: Clinical features and results of treatment in 4 patients. Surg Oncol 2018;27:657-62.

10. Mangan C, Moinuddin Z, Summers A, et al. Encapsulating peritoneal sclerosis following hyperthermic intraperitoneal chemotherapy. ANZ J Surg 2019;89:E468-9.

11. Vlasveld LT, Taal BG, Kroon BB, et al. Intestinal obstruction due to diffuse peritoneal fibrosis at 2 years after the successful treatment of malignant peritoneal mesothelioma with intraperitoneal mitoxantrone. Cancer Chemother Pharmacol 1992;29:405-8.

12. Danford CJ, Lin SC, Smith MP, et al. Encapsulating peritoneal sclerosis. World J Gastroenterol 2018;24:3101-11.

13. Narayanan R, Bhargava BN, Kabra SG, et al. Idiopathic sclerosing encapsulating peritonitis. Lancet 1989;2:127-9.

14. Devay AO, Gomceli I, Korukluoglu B, et al. An unusual and difficult diagnosis of intestinal obstruction: The abdominal cocoon. Case report and review of the literature. World J Emerg Surg 2008;3:36.

15. Tu JF, Huang XF, Zhu GB, et al. Comprehensive analysis of 203 cases with abdominal cocoon. Zhonghua Wei Chang Wai Ke Za Zhi 2006;9:133-5.

16. Triga K. Encapsulating peritoneal sclerosis: common or rare in peritoneal dialysis? Saudi J Kidney Dis Transpl 2013;24:223-9.

17. Brown EA, Bargman J, van Biesen W, et al. Length of Time on Peritoneal Dialysis and Encapsulating Peritoneal Sclerosis - Position Paper for ISPD: 2017 Update. Perit Dial Int 2017;37:362-74.

18. Eltringham WK, Espiner HJ, Windsor CW, et al. Sclerosing peritonitis due to practolol: a report on 9 cases and their surgical management. Br J Surg 1977;64:229-35.

19. Sarker S, Kodali S, Weber F. A new meaning to butterflies in the stomach. Gastroenterology 2015;148:e12-3.

20. Sharma V, Mandavdhare HS, Rana SS, et al. Role of conservative management in tubercular abdominal cocoon: a case series. Infection 2017;45:601-6.

21. Simbli MA, Niaz FA, Al-Wakeel JS. Encapsulating peritoneal sclerosis in a peritoneal dialysis patient presenting with complicated Mycobacterium fortuitum peritonitis. Saudi J Kidney Dis Transpl 2012;23:635-41.

22. Sigaroudinia MO, Baillie C, Ahmed S, et al. Sclerosing encapsulating peritonitis--a rare complication of ventriculoperitoneal shunts. J Pediatr Surg 2008;43:E31-3.

23. Hsu YH, Hsia CC, Tsai DM, et al. Development of encapsulating peritoneal sclerosis following bacterial peritonitis in a peritoneal dialysis patient. Am J Kidney Dis 2010;55:198-202.

24. Pepels MJ, Peters FP, Mebis JJ, et al. Sclerosing peritonitis: an unusual cause of ascites in a patient with systemic lupus erythematosus. Neth J Med 2006;64:346-9.

25. Dabak R, Uygur-Bayramicli O, Aydin DK, et al. Encapsulating peritonitis and familial Mediterranean fever. World J Gastroenterol 2005;11:2844-6. 
26. Honda K, Oda H. Pathology of encapsulating peritoneal sclerosis. Perit Dial Int 2005;25 Suppl 4:S19-29.

27. Alston H, Fan S, Nakayama M. Encapsulating Peritoneal Sclerosis. Semin Nephrol 2017;37:93-102.

28. Markman M, Cleary S, Howell SB, et al. Complications of Extensive Adhesion Formation after Intraperitoneal Chemotherapy. Surg Gynecol Obstet 1986;162:445-8.

29. Jacquet P, Sugarbaker PH. Abdominal adhesions causing intestinal obstruction following cytoreductive surgery and early postoperative intraperitoneal chemotherapy. Acta Chirurgica Austriaca 1995;27:92-5.

30. Zhang CH, Yu JW, Luo M. Multicystic peritoneal mesothelioma: A short review. Curr Probl Cancer 2017;41:340-8.

Cite this article as: Ni Z, Chen Q, Huang C, Wang S, Huang Q. Sclerosing encapsulating peritonitis as a rare cause of intestinal obstruction after the treatment of peritoneal mesothelioma: a case report and review of the literature. Transl Cancer Res 2021;10(6):3074-3080. doi: 10.21037/tcr-20-3259
31. Kanai G, Kakuta T, Hirukawa T, et al. A Case of Encapsulating Peritoneal Sclerosis Complicated by Malignant Peritoneal Mesothelioma. Tokai J Exp Clin Med 2016;41:135-8.

32. Wei B, Wei HB, Guo WP, et al. Diagnosis and treatment of abdominal cocoon: a report of 24 cases. Am J Surg 2009;198:348-53.

33. Li Y, Li N, Zhu WM, et al. Surgical treatment for idiopathic abdominal cocoon. Zhonghua Wai Ke Za Zhi 2013;51:139-41.

34. Lim MC, Chotai NC, Giron DM. Idiopathic Sclerosing Encapsulating Peritonitis: A Rare Cause of Subacute Intestinal Obstruction. Case Rep Med 2016;2016:8206894. 